\title{
Integrated Magnetic Full Wave Converter With Flexible Output Inductor
}

\author{
Liang Yan, Student Member, IEEE, Dayu Qu, Member, IEEE, and Brad Lehman, Member, IEEE
}

\begin{abstract}
A new integrated magnetic full wave dc/dc converter ${ }^{1}$ that provides flexible transformer design by incorporating an independent output inductor winding is introduced. The transformer is implemented on a traditional three-leg magnetic core. The inductor winding can be separately designed to control the output current ripple. The cross-sectional area of the inductor core leg can be reduced dramatically. The operation and performance of the proposed circuit are verified on a $100 \mathrm{~W}$ prototype converter.
\end{abstract}

Index Terms-Current doubler, dc-dc converter, full wave topology, integrated magnetics.

\section{INTRODUCTION}

I NTEGRATED magnetic techniques combine transformers and inductors into a single core in order to reduce the size and cost of magnetic components in $\mathrm{dc} / \mathrm{dc}$ converters. Principles of modeling and designing integrated magnetics have been widely published [3]-[10]. Historically, integrated dc/dc converters were first developed for Forward converters [3], [4]. However, recently integrated magnetic full wave circuits have been extensively studied [11]-[13]. These full wave circuits have reported significant benefits, including high efficiency, high power density and low switch stress. Applications of these circuits are being proposed in $30 \mathrm{~W} \sim 100 \mathrm{~W}$ range, and have great potential for telecommunication power supplies.

A "typical" integrated magnetic full wave dc/dc converter is shown in Fig. 1 [13], which uses a three-leg magnetic core. This class of full wave integrated magnetic circuits exhibits a common character that there is no winding on the inductor leg. Instead, the secondary windings play the role of the equivalent inductor winding, and hence, the secondary windings must satisfy both power transfer and current ripple restrictions for different operating stages.

The elimination of the inductor winding comes from the magnetic integration methods [11], and, in applications, this is believed to reduce the copper loss and simplify the transformer structure. However, optimum transformer design must keep the

Manuscript received February 5, 2000; revised November 1, 2002. This paper was presented in part at the IEEE Applied Power Electronics Conference (APEC); Dallas, TX, March 2002 [1]. Furthermore, an identical circuit was independently developed and presented by J. Sun, et al. at IEEE APEC, March 2002 [2]. Recommended by Associate Editor K. Ngo.

The authors are with Department of Electrical and Computer Engineering, Northeastern University, Boston, MA 02115 USA (e-mail: lyan@ece.neu.edu; lehman@ece.neu.edu).

Digital Object Identifier 10.1109/TPEL.2003.809357

${ }^{1}$ Patent pending.

${ }^{2}$ This paper refers to an integrated magnetic full wave circuit as being "typical" or "conventional" only in the sense that there is no winding on the inductor leg.

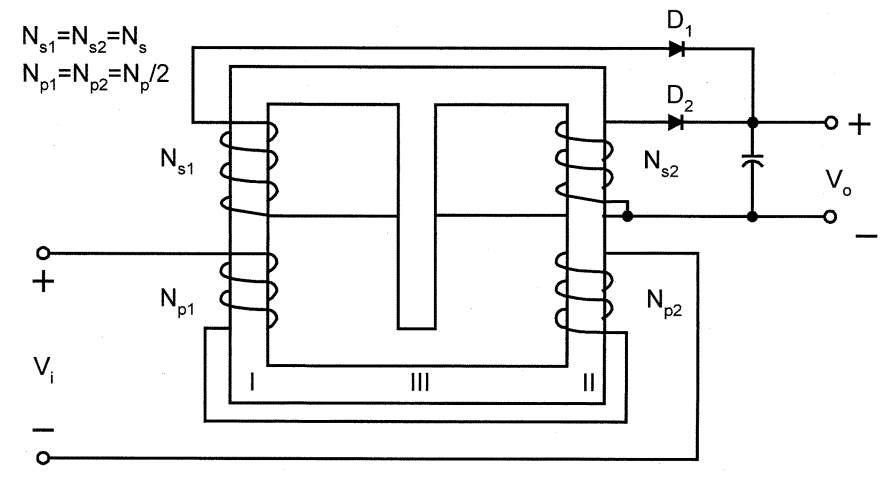

Fig. 1. Conventional integrated magnetic full wave circuit.

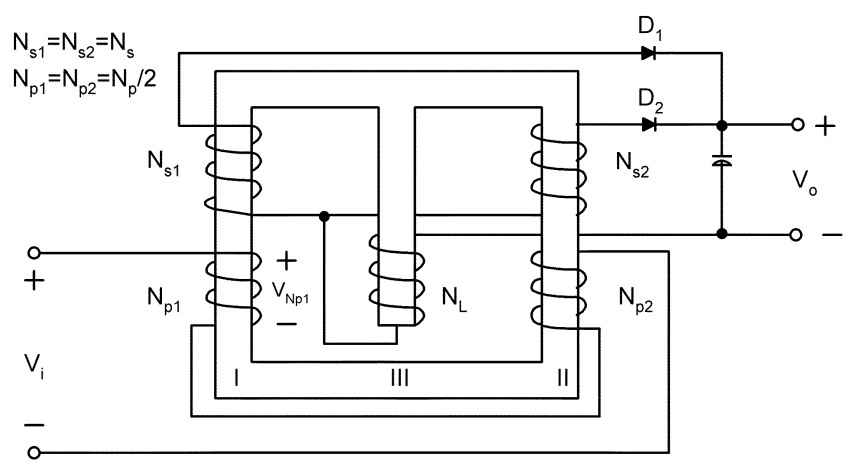

Fig. 2. Proposed integrated magnetic full wave circuit (Structure I).

balance between copper loss, ferrite loss and total size. In integrated magnetic transformers, such as those shown in Fig. 1, the number of secondary winding turns influences the number of primary winding turns, the magnetic core size and the output current characteristics. This places a burden on the secondary winding turn decision and complicates the transformer design. As a result, the magnetic core often needs to be specially designed and manufactured in order to satisfy both output current ripple requirements and flux density constraints.

This paper presents a new integrated magnetic full wave converter, as shown in Fig. 2. In this full wave circuit, the inductor winding is retained and can be designed independently from the secondary windings. The added flexibility allows the use of commercial magnetic cores, and the transformer design can be optimized. Specifically, the circuit has the following characteristics.

i) Retains the original full wave buck mode circuit operation.

ii) Integrates an inductor winding into the transformer. 


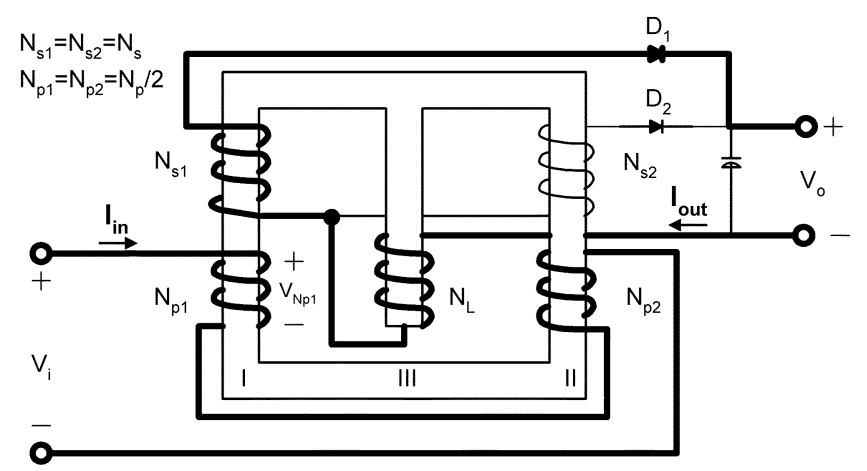

(a)

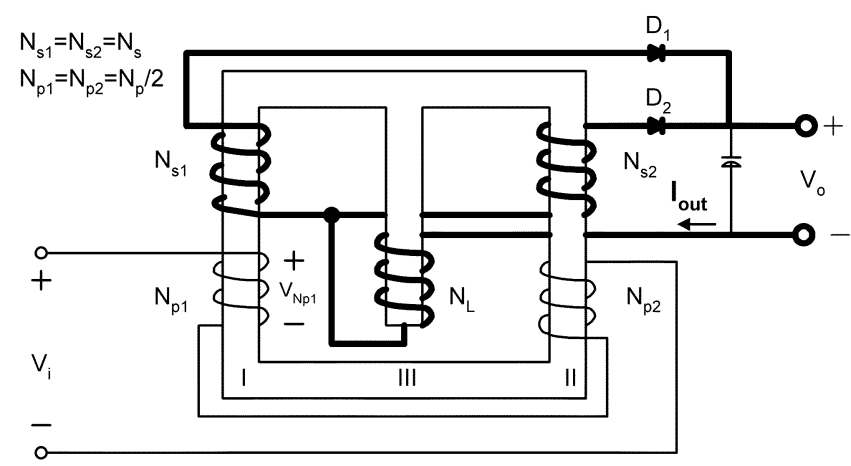

(b)

Fig. 3. Operation modes.

iii) The inductor winding does not appear in the input-tooutput voltage transfer function.

iv) The output current is continuous.

v) The inductor can be designed specifically to satisfy the current ripple restriction and the magnetic constraints.

vi) The cross-sectional area of the inductor leg can be much smaller than that of the conventional integrated magnetic circuit to achieve the same output current ripple.

vii) This circuit has been reported by [2] to achieve $92.9 \%$ power efficiency at $100 \mathrm{~W}$ for a half-bridge dc/dc converter on an E22 core and a 3 in $\times 2.74$ in circuit board. (However, in this paper we present a simple and rudimentary full-bridge prototype, for proof of concept purposes only, which achieves around $90 \%$ power efficiency.)

Section II presents the operation principle of the proposed circuit. Section III introduces the transformer design approach. Section IV provides some character comparisons between the conventional and the proposed circuit. An alternative topology of the proposed converter is also introduced. Section V shows the simulation and experimental results for a design example. Section VI gives the conclusion. Details of the transformer design are presented in the Appendix.

\section{OPERATION PRINCIPLE}

The basic operation principle of the proposed integrated magnetic full wave circuit in Fig. 2 is similar to the conventional integrated magnetic full wave circuit in Fig. 1. The new converter has two basic operating modes within each half-cycle. The current paths in each mode are illustrated in Fig. 3. In Fig. 4, the magnetic states within each core leg are modeled by using the capacitive modeling method [7]-[10].

The capacitive modeling method is briefly described here. In this model, each current source or sink represents an active winding; $\dot{\Phi}$ is the flux rate (i.e. the derivative of flux) within each leg; $F$ is the magnetomotive force on the permeance; $P$ is the permeance of the gap or each core leg. For a winding on a core leg, two basic relations provide the interface between the magnetic circuit and the electrical circuit

$$
\begin{aligned}
\dot{\Phi} & =\frac{V}{N} \\
F & =N I
\end{aligned}
$$

$\mathrm{KCL}$ and KVL rules control the variables in the magnetic circuit. Gap is abstracted as permeance $P_{g}$

$$
P_{g}=\mu_{0} \frac{A}{l}{ }_{g}
$$

where

$V \quad$ voltage on the winding;

$N$ number of winding turn;

$I \quad$ current in the winding;

$\mu_{0} \quad$ permeability of the air;

A cross-sectional area of the core leg with gap;

$l_{g} \quad$ gap length.

Fig. 5 illustrates the typical operating waveforms. To simplify the analysis, all the devices are assumed to be ideal. The magnetomotive forces on the permeances of the core legs are neglected, i.e. these permeances are assumed to be infinite compared with the permeance of the gap. The leakage energy, which is a major implementation problem for most integrated magnetic converters, is not considered at this moment. Let $V_{i}$ be as in Fig. 5. The operation principle is explained as follows.

Mode I: The input voltage is $V_{i}>0$. The primary winding initiates the flux rates $\dot{\Phi}_{p 1}$ and $\dot{\Phi}_{p 2}$. The voltages activated on the secondary windings force the rectifier $D_{1}$ to conduct. The current on the secondary side flows through $N_{L}, N_{s 1}, D_{1}$ and the load. The flux rate difference between LegI and LegII causes flux accumulation in the center leg, which increases the energy storage within the gap. The flux transitions for each leg (I, II, and III) are shown in Fig. 5, where every flux $\Phi$ has the same polarity as its corresponding $F$ and $\Phi=F P$.

From Fig. 3 and Fig. 4, the flux rates in each leg can be determined from the magnetic path and the outer circuit. Assume the voltage drop on winding $N_{p 1}$ is $V_{N p 1}$ then, in the magnetic circuit

$$
\begin{aligned}
\dot{\Phi}_{p 1} & =\frac{V_{N p 1}}{N_{p 1}} \\
\dot{\Phi}_{p 2} & =\frac{V_{i}-V_{N p 1}}{N_{p 2}} \\
\dot{\Phi}_{L_{I}} & =\dot{\Phi}_{p 2}-\dot{\Phi}_{p 1}
\end{aligned}
$$

$\dot{\Phi}_{L_{-} I}$ is also controlled by winding $N_{L}$ and can be represented as

$$
\dot{\Phi}_{L \_I}=\frac{\dot{\Phi}_{p 1} N_{s}-V_{o}}{N_{L}} .
$$




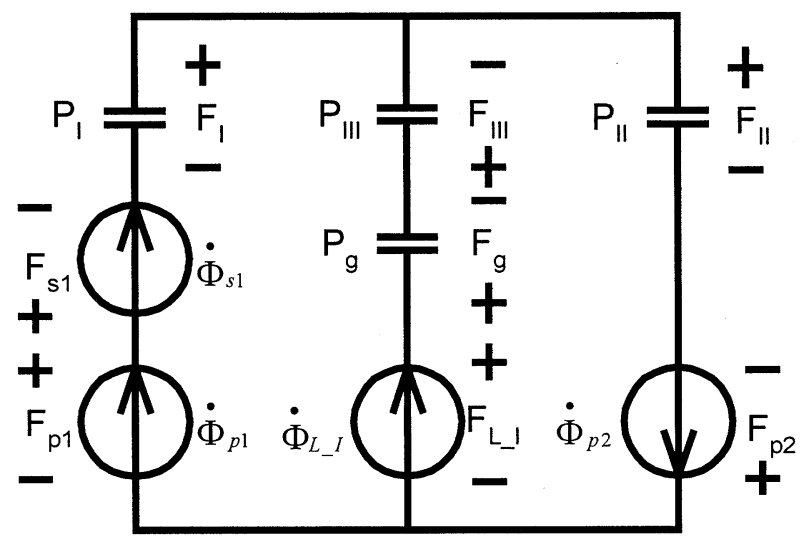

Fig. 4. Analysis models.

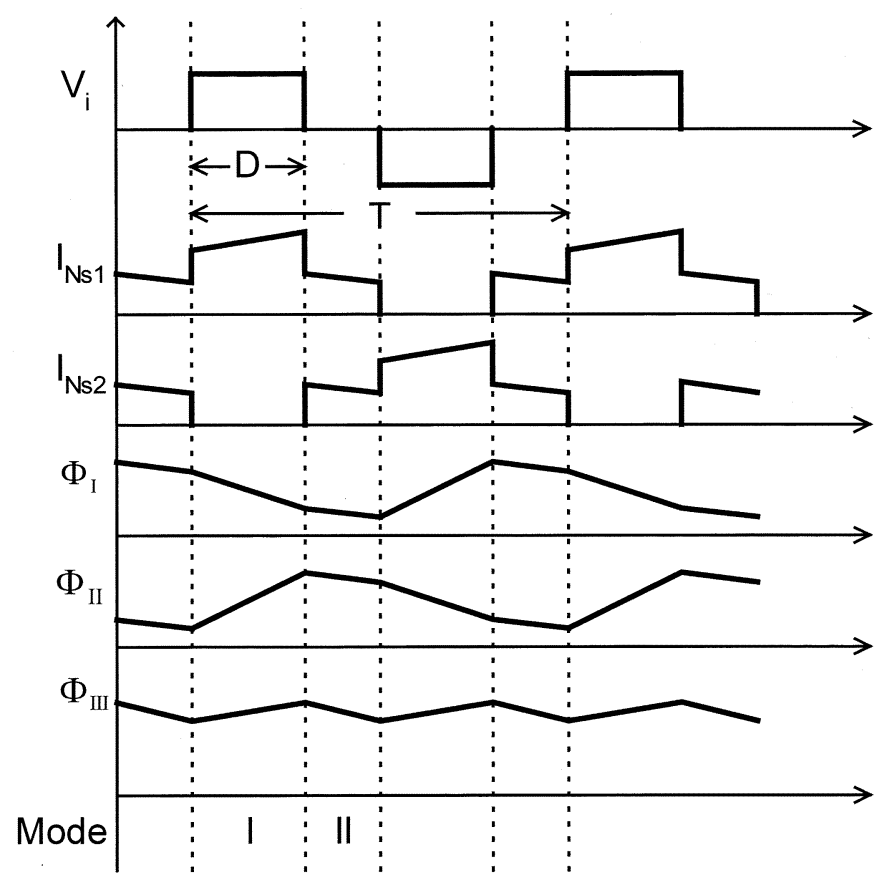

Fig. 5. Operation waveforms of the new integrated magnetic full wave circuit.

If $N_{p 1}=N_{p 2}=N_{p} / 2$, the flux rate in the center leg can then be obtained from (4)-(7)

$$
\dot{\Phi}_{L I}=\frac{\left(N_{s} / N_{p}\right) V_{i}-V_{o}}{N_{L}+N_{s} / 2}
$$

where

$N_{p} \quad$ total primary winding turn on $\operatorname{LegI}$ and $\operatorname{LegII}$;

$N_{s} \quad$ secondary winding turn on $\operatorname{LegI}$ and $\operatorname{LegII}$;

$N_{L} \quad$ inductor winding turn on LegIII;

$V_{i} \quad$ input voltage;

$V_{o} \quad$ desired output voltage.

Equation (8) indicates that the flux rate is constant. So the magnetomotive force on the gap $F_{g}$ increases linearly. So, referring to (26) in the Appendix, the current $I_{L}$ in $N_{L}$, or equivalently, the current $I_{s 1}$ in $N_{s 1}$, increases linearly as in Fig. 5.

Mode II: The input voltage is $V_{i}=0$. The primary winding is free. The energy within the gap will release and the entire

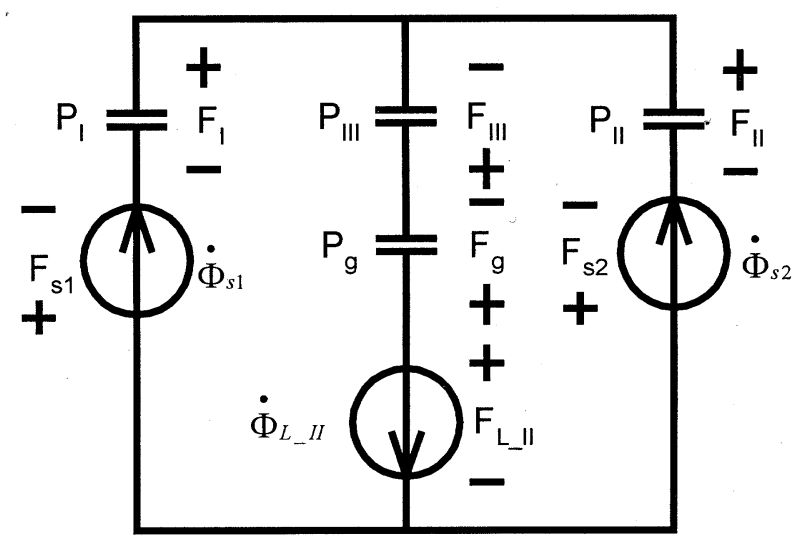

transformer behaves as an inductor. Both the secondary windings conduct. The two secondary output current paths share the inductor winding. The magnetic state of the transformer is symmetric along the center leg. The flux in the center leg decreases.

Applying the same derivation method as in Mode I, the flux rate in the center leg is obtained

$$
\dot{\Phi}_{L \_I I}=\frac{V_{o}}{N_{L}+\frac{N_{s}}{2}} .
$$

In the next half-cycle, Mode I appears for the winding on the other side. Mode II is exactly the same as in this half-cycle. To derive the input-to-output voltage transfer relation, suppose the duty ratio is equal to $D(D<0.5)$ as in Fig. 5. Since the flux change should be balanced on the gap in each half cycle

$$
\dot{\Phi}_{L_{-} I} D=\dot{\Phi}_{L_{-} I I}(0.5-D) .
$$

From (8)-(10), the ideal voltage transfer function is

$$
\frac{V_{o}}{V_{i}}=2 D \frac{N_{s}}{N_{p}} .
$$

Note that the inductor winding $N_{L}$ is not included in (11). So, the design of input-to-output voltage transfer ratio and the output current ripple of the integrated magnetic transformer can be separated as in a typical discrete core full wave buck mode circuit.

Equation (9) indicates that the flux rate is constant. The magnetomotive force $F_{g}$ on the gap decreases linearly. So, the current $I_{L}$ in $N_{L}$ decreases linearly. The currents in the two secondary windings are half of the current in the inductor winding, shown in Fig. 5.

\section{DESIGN APPROACH}

Transformer design is vital to achieve favorable performance in any converter, especially for a circuit with integrated magnetics, which is based on the tradeoff between output current ripple, flux density and magnetic core size. For the design in the newly proposed topology, the following constraints are set in advance.

1) Commercial magnetic cores are available for selection.

2) Tolerable output current ripple is specified.

3) Allowable peak flux density in the core legs is defined. 
The purpose of the design is to select the transformer parameters to satisfy the electrical and the magnetic specifications. Specifically, these parameters include

1) magnetic core type and size;

2) total primary winding turn $N_{p}=2 N_{p 1}=2 N_{p 2}$;

3) secondary winding turn $N_{s}=N_{s 1}=N_{s 2}$;

4) inductor winding turn $N_{L}$.

In the design, the deterministic parameters are calculated first. Then, the combination of flexible variables is selected. The design may follow three steps.

Step 1) Select the primary-to-secondary winding turns ratio $n=N_{p} / N_{s}$.

The primary-to-secondary winding turns ratio is determined by the input and output voltage specification from (11). Normally, $n$ is selected to be as large as possible to use the duty ratio more efficiently and to reduce the number of secondary winding turns. However, the line loss and the maximum duty ratio of the controller should be considered to achieve full range input voltage operation.

Step 2) Select the magnetic core.

Since the commercial magnetic core is used, it is selected from the available core list according to the output power specification.

Step 3) Select the winding turns.

The voltage transfer function is only determined by $n$. Once $n$ is selected, the winding turns are determined by the transformer constraints. The step is to select $N_{s}$ and $N_{L}$. This may follow the well-established traditional transformer design methods, either by trial and error or by acquiring the optimum combination from the entire parameter lists. The important consideration is to calculate the output current ripple and the peak flux density. The following formulas can be used to verify that these results are within the tolerance. The derivation of the formulas is presented in the Appendix.

1. Verify that the output current ripple is within the design specification.

The maximum peak-to-peak output current ripple can be derived as

$$
I_{L \_d}=\frac{V_{o}\left(0.5-D_{\min }\right)}{f_{s} P_{g}\left(N_{L}+\frac{N_{s}}{2}\right)^{2}}
$$

where

$f_{s} \quad$ switching frequency;

$D_{\text {min }}$ minimum duty ratio.

2. Verify that the peak flux density in the magnetic core is below the saturation value.

The average flux density in the center leg is

$$
B_{c \_a v}=\frac{N_{L}+\frac{N_{s}}{2}}{A_{c}} I_{L \_a v P g}
$$

where

$I_{L_{\text {a av }}}$ average output current;

$A_{c} \quad$ cross-sectional area of the center leg.
The average flux density in the outer legs is (identical in the two legs)

$$
B_{O \_a v}=\frac{B_{c \_a v}}{2}
$$

The maximum flux swing in the center leg is

$$
B_{c_{-} d}=\frac{\left(N_{L}+\frac{N_{s}}{2}\right) I_{L_{-} d} P_{g}}{A_{c}} .
$$

The maximum flux swing in the outer legs is

$$
B_{o \_d}=\frac{V_{o}\left(\frac{N_{L}}{N_{s}}+1-D_{\min }\right)}{2 f_{s} A_{o}\left(N_{L}+\frac{N_{s}}{2}\right)}
$$

where, $A_{o}$ : the cross-sectional area of the outer legs. The peak flux density in the center leg is

$$
B_{c_{-} p}=B_{c_{-} a v}+\frac{B_{c_{-} d}}{2} .
$$

The peak flux density in the outer legs is

$$
B_{o_{-} p}=B_{o_{-} a v}+\frac{B_{o_{-} d}}{2} .
$$

Both $B_{c_{-} p}$ and $B_{O_{-} p}$ must be below the saturation value.

The above design approach is based on ideal magnetic characteristics, which assume the following.

1) The permeability of magnetic core is infinite.

2) The B-H curve is linear.

3) The stray capacitance and the leakage inductance are not considered.

Because of the infinite permeability assumption, the magnetomotive force in the core is neglected compared with that of the gap. However, because of this ideal assumption, the current ripple will actually be larger than the above design value. On the other hand, this also implies that the average flux density in the center leg is smaller than the ideal case, since the average flux produced from the average output current is divided between the gap and the core leg. After the design, the finite permeability effect can be considered from simulation directly [7], [10].

The nonlinear B-H curve requires conservative design for peak flux density. As the flux goes higher, the effective permeability is lower. The reduced permeance leads to larger current ripple. A typical phenomenon is that the current waveform is no longer piecewise triangular, but exhibits nonlinear characteristics.

Stray capacitance and leakage inductance are major parasitic parameters in magnetic components. They cause ringing on switches and rectifiers. It is, therefore, important to have tight coupling of the transformer windings. Section V also discusses an electrical solution to this problem. Since the proposed circuit can reduce the primary and secondary winding turns, it should have smaller stray capacitance and leakage inductance than the conventional circuit.

With the knowledge of average flux density and flux density swing, it is also possible to estimate the power loss from available models [14], [15]. These extensive calculations are not included in this paper due to their length. 


\section{TOPOLOGY COMPARISONS}

This section compares the previously known integrated magnetic full wave topology in Fig. 1 with the proposed circuit in Fig. 2. An alternative structure of the proposed circuit is also introduced.

\section{A. Conventional Topology Versus Proposed Topology}

1) Structure Difference: Compared with conventional integrated magnetic full wave topologies (see Fig. 1), the proposed topology has an additional inductor winding on the center leg. It is obvious that when the inductor winding turn is equal to zero $\left(N_{L}=0\right)$, the proposed circuit is the same as that in Fig. 1. From this point of view, the circuit in Fig. 1 is a special case of the proposed topology.

2) Design Considerations: The transformer design for the new circuit has the flexibility to select the number of inductor winding turns, which can vary from zero to any reasonable number. When the inductor winding turn is small, the secondary winding turn has to be large enough to reduce the output current ripple according to (12). Because the primary-to-secondary turns ratio $n$ is fixed, the large secondary winding turn leads to large primary winding turn. Both of them cause more copper loss and may also be hard to fit into the magnetic core. On the other hand, it is true that the inductor leg of the magnetic core can be specifically designed to achieve the same performance by large cross-sectional area. However, this increases the cost of designing and manufacturing the specialized magnetic core. At the same time, the additional ferrite volume also increases the ferrite loss, especially in high frequency applications.

The effect of the inductor winding can be further investigated from (3), (12), and (13). It is easy to find that $B_{c_{-} a v} \propto\left(\left(N_{L}+\right.\right.$ $\left.\left.\left(N_{s} / 2\right)\right) / l_{g}\right)$ and $I_{L_{-} d} \propto\left(l_{g} /\left(A_{c}\left(N_{L}+N_{s}\right)^{2}\right)\right)$. The winding turn $N_{L}+\left(N_{s} / 2\right)$ is proportional to $B_{c_{-} a v}$ However, it is a quadratic factor to reduce the current ripple. So, if both $N_{L}+$ $\left(N_{s} / 2\right)$ and $l_{g}$ are doubled, while maintaining the output current ripple and the average flux density, the cross-sectional area $A_{c}$ can be decreased to half.

An example of quantitative comparisons and choice of inductor winding turns is shown in Section V.

\section{B. Alternative Structure of Proposed Circuit}

1) Structure Comparison: Fig. 6 shows an alternative structure (Structure II) of the proposed circuit. The connection of the inductor winding is reversed compared with Structure I in Fig. 2. The circuit characteristics can be derived by using the same analysis method.

2) Characteristics Comparison: The operation principle of Structure II is the same as Structure I. The inverse connection of the inductor winding does not change the voltage transfer function. However, the magnetic states within the core are different because the magnetizing direction of the center leg of Structure II is reversed too.

In operation Mode I, primary winding $N_{p 2}$ of Structure I provides both the output power through the secondary winding $N_{s 1}$ and the stored energy in the gap. However, in Structure II it is the opposite primary winding $N_{p 1}$ that provides the energy.

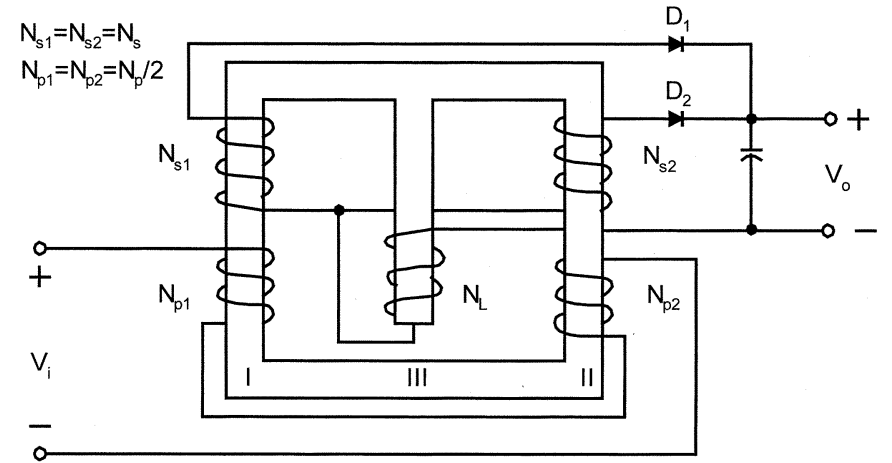

Fig. 6. Alternative structure of proposed integrated magnetic full wave circuit (Structure II).
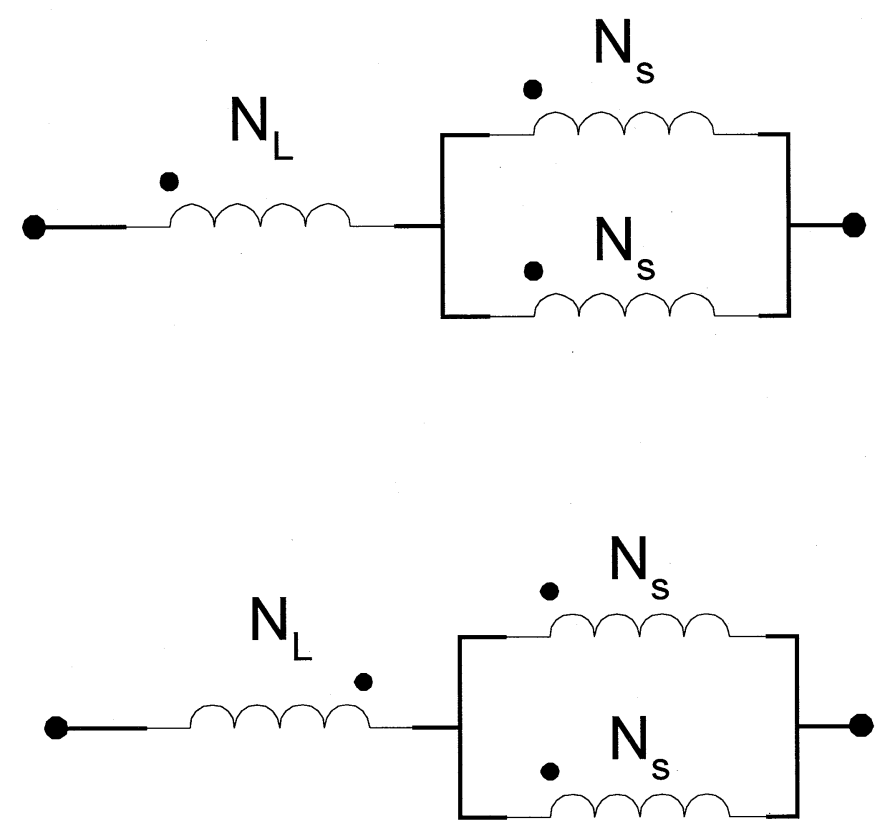

Fig. 7. Equivalent inductor turn. Upper: structure I. Lower: structure II.

In operation Mode II, these two circuits exhibit different equivalent inductor winding connections as shown in Fig. 7. The equivalent inductor turns are

$$
\begin{array}{cl}
\text { Structure I } & N_{L}+\frac{N_{s}}{2} \\
\text { Structure II } & N_{L}-\frac{N_{s}}{2} \quad\left(N_{L} \geq \frac{N_{s}}{2}\right) .
\end{array}
$$

If comparing the integrated magnetic full wave circuit in Fig. 1 with Structure II, the former is equivalent to the latter when $N_{L}=N_{s}$ This can be verified by replacing $N_{L}$ with zero in (19) and replacing $N_{L}$ with $N_{s}$ in (20). Both lead to the same equivalent inductor turn $N_{s} / 2$, which is also the equivalent inductor turn of the conventional circuit.

From another point of view, if "negative winding turn" is allowed, the two proposed structures could be combined into one form as in Fig. 2. The inductor winding turn $N_{L}$ is negative if the connecting direction is reversed. Then the equivalent inductor turns would be (19) with restriction that $N_{L} \neq-\left(N_{s} / 2\right)$. 


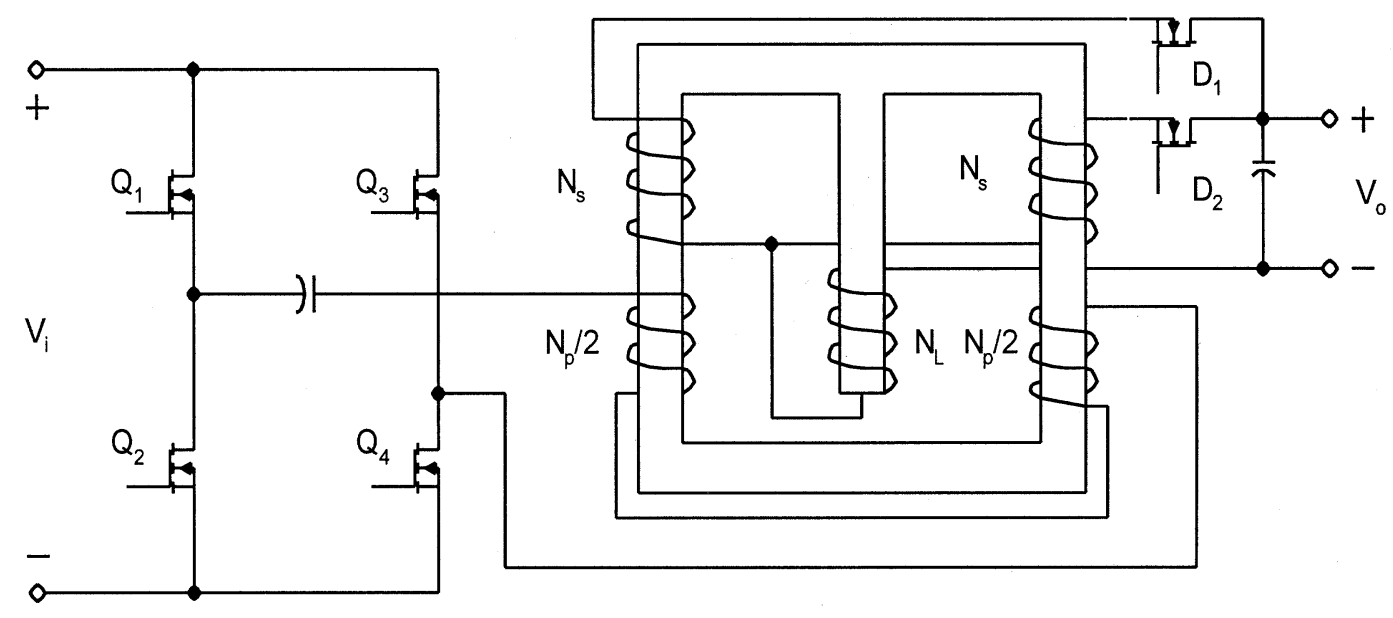

Fig. 8. Experimental circuit.

\section{SimUlation AND EXPERIMENTAL RESUlTS}

A $100 \mathrm{~W} / 3.3 \mathrm{~V}, 150 \mathrm{kHz}$ full bridge dc/dc prototype module is designed and built to verify the analysis. The circuit is shown in Fig. 8 and the design specifications are shown in Table I. Fig. 8 uses the full-bridge configuration only as an example. The addition of inductor winding turn can be applied to other topologies, such as half-bridge, push-pull, although the circuit design may be reconsidered.

\section{A. Design}

The primary-to-secondary winding turns ratio can be calculated as $n=9$. Commercial E22/A160 core is selected. Table II compares the magnetic constraint values, where the inductor winding $N_{L}$ varies from zero to three, and the secondary winding turn $N_{s}$ varies from two to four. It is obvious that when $N_{L}=0$, only $N_{s}=4$ leads to the tolerable output current ripple. This is the case of the conventional integrated magnetic circuit. However, the primary winding turn will be $N_{p}=36$. As Table II shows, a reasonable choice of the inductor winding turn is one, i.e. $N_{L}=1$. Further increase of the inductor winding turn will not reduce the current ripple much and the flux density will be much higher. The secondary winding turn can be two or three. To reduce the loss and size, two-turn is a better choice. So, the winding turns are determined

$$
N_{p 1}=N_{p 2}=\frac{N_{p}}{2}=9, N_{s}=2, N_{L}=1 .
$$

The constraint values are tolerable in a $100 \mathrm{~W} \mathrm{dc/dc} \mathrm{module.}$

Output current ripple $I_{L_{-} d}=10.4 \mathrm{~A}$.

Peak flux density in the center leg $B_{c_{-} p}=144 \mathrm{mT}$.

Peak flux density in the outer leg $B_{o_{-} p}=154 \mathrm{mT}$.

Further comparison with the conventional integrated magnetic transformer design shows that for the same E22 core, the same output current ripple, and the same primary and secondary winding turns, the flux densities for the conventional circuit are twice the value of the newly proposed circuit. This could cause higher core loss due to the increase of core loss coefficient. If both the primary and the secondary winding turns are increased, the flux densities in the conventional circuit can be reasonable. However, the added copper size is larger than the
TABLE I

CiRCUIT SPECIFICATIONS

\begin{tabular}{c|c}
\hline Input Voltage & $36 \mathrm{~V} \sim 75 \mathrm{~V}$ \\
\hline Output Voltage & $3.3 \mathrm{~V}$ \\
\hline Maximum Output & $100 \mathrm{~W}$ \\
\hline Switching Frequency & $150 \mathrm{kHz}$ \\
\hline
\end{tabular}

TABLE II

TRANSFORMER DESIGN CONSTRAINTS From DIFFERENT WINDING TURNS

\begin{tabular}{l|c|c|c|c}
\hline & $\mathrm{N}_{\mathrm{L}}=0$ & $\mathrm{~N}_{\mathrm{L}}=1$ & $\mathrm{~N}_{\mathrm{L}}=2$ & $\mathrm{~N}_{\mathrm{L}}=3$ \\
\hline $\mathrm{N}_{\mathrm{S}}=2$ & 41.7354 & 10.4339 & 4.6373 & 2.6085 \\
\hline $\mathrm{N}_{\mathrm{S}}=3$ & 18.5491 & 6.6777 & 3.4070 & 2.0610 \\
\hline $\mathrm{N}_{\mathrm{S}}=4$ & 10.4339 & 4.6373 & 2.6085 & 1.6694 \\
\hline
\end{tabular}

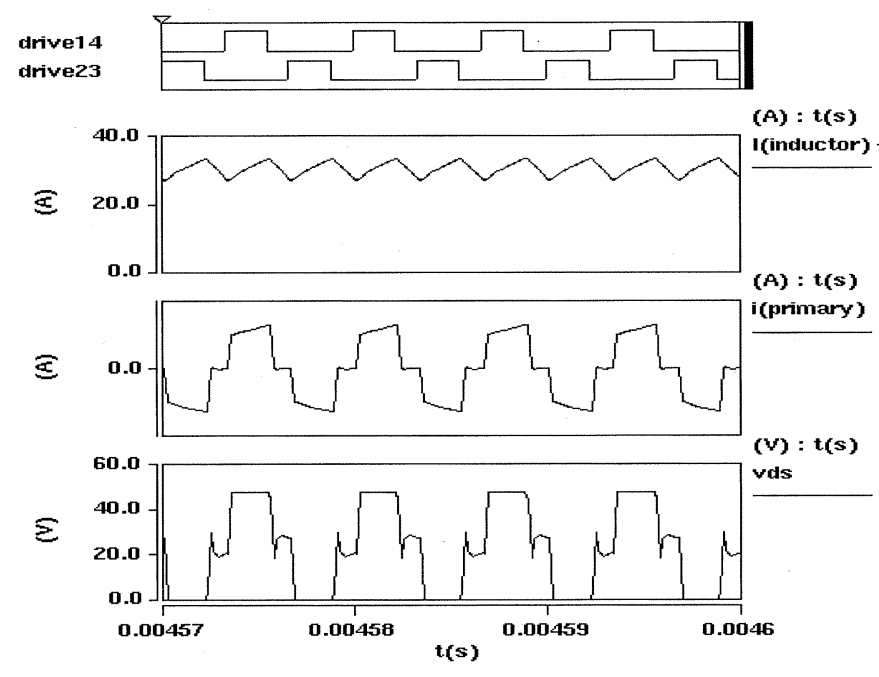

Fig. 9. Simulation results.

new circuit. Of course, the new circuit has a disadvantage of inductor copper loss compared to the circuit in Fig. 1. So there are 


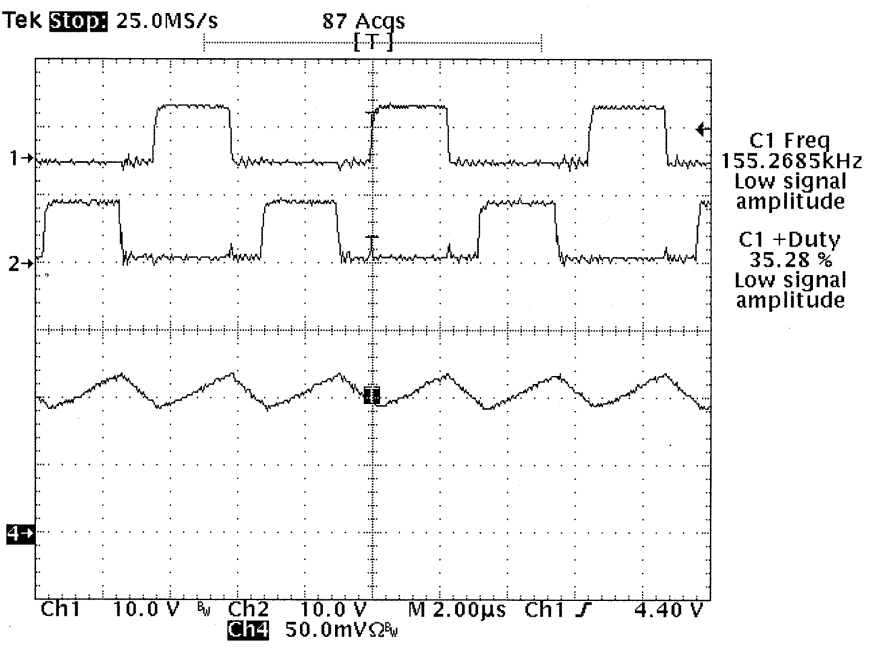

Fig. 10. Inductor current (Ch 1, Ch 2: Driving signal; Ch4: Inductor current).

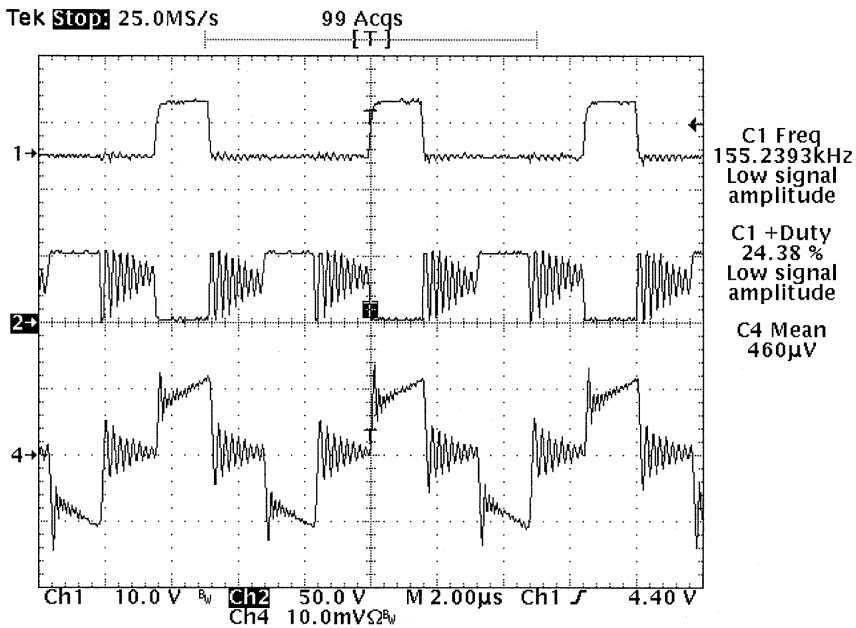

Fig. 11. Primary current and $\mathrm{V}_{\mathrm{ds}}$ of switch (Ch1: Driving signal; $\mathrm{Ch} 2$ : $\mathrm{V}_{\mathrm{ds}}$ of the primary switch; Ch 4 : Primary current).

design trade-offs. Both circuits in Fig. 1 and Fig. 2 have corresponding advantages and disadvantages.

\section{B. Simulation}

In Fig. 9, drive 14 and drive 23 are the driving signal of the switches on the primary side. $\mathrm{I}_{\text {primary }}$ is the current waveform in the primary winding. $I_{\text {inductor }}$ is the current waveform in the inductor winding. $\mathrm{V}_{\mathrm{ds}}$ is the drain-source voltage waveform on the primary switches. This figure shows the operation when the input voltage is $48 \mathrm{~V}$ and the output current is $30 \mathrm{~A}$.

\section{Experiment}

To build the circuit, a Philips 3F3 E22/6/16 planar core is selected. Four Si4480 MOSFET's are used as the primary switches. Three parallel Si4466 synchronous rectifiers are used for each rectification path on the secondary side. The secondary stage uses the controlled synchronous rectification scheme [16], [17]. To keep the flux balance, an isolation capacitor is in series with the primary winding of the transformer.

Fig. 10 is the experimental result of the inductor current. Fig. 11 shows the experimental drain-source voltage waveform

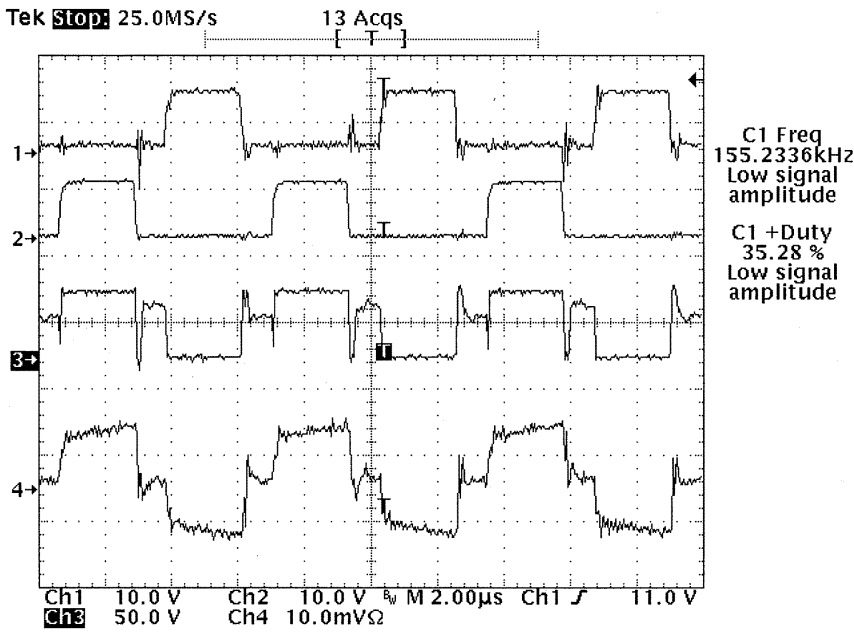

Fig. 12. Primary current and $\mathrm{V}_{\mathrm{ds}}$ of switch with active leakage energy recovery circuit $\left(\mathrm{Ch} 1, \mathrm{Ch} 2\right.$ : Driving signal; $\mathrm{Ch} 3: \mathrm{V}_{\mathrm{ds}}$ of the primary switch; Ch4: Primary current).

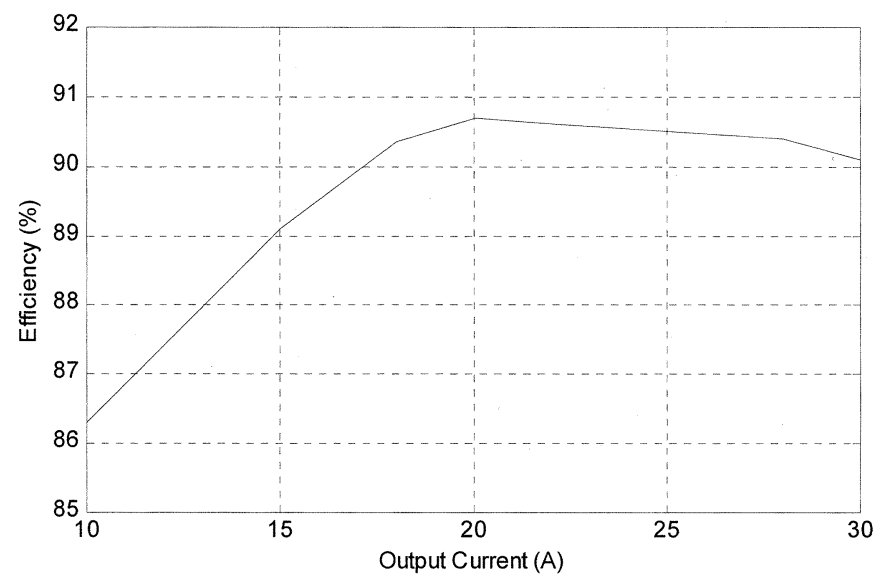

Fig. 13. Total efficiency versus output current (input voltage 48V / output voltage $3.3 \mathrm{~V}$ )

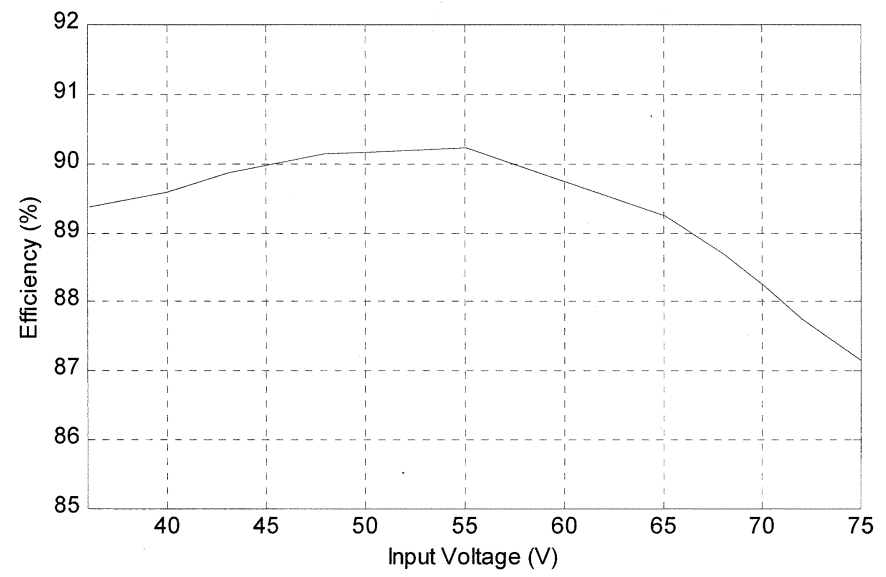

Fig. 14. Total efficiency versus input voltage (output current 30 A / output voltage $3.3 \mathrm{~V}$ ).

of the primary switch and the current waveform in the primary winding. The results verify the analysis. It also shows that the ringing exists due to the leakage inductance. To eliminate the ringing, either phase shift technique or additional active clamp 
circuit can be used. Fig. 12 shows the result by using an active clamp circuit. The waveform is clean. However, in low power dc/dc converters, the power loss in the additional circuit is often larger than the loss from the ringing. Furthermore, in a full bridge circuit, the voltage on primary switches is naturally clamped by input voltage. The circuit works safely without the clamp circuit and achieves the efficiency as shown in Fig. 13 and Fig. 14. It is important to point out that the experiments in this paper focus on proof-of-concept for the operating principle. In [2], by using superior manufacturing techniques, $92.9 \%$ power efficiency is achieved for a $100 \mathrm{~W}$, half-bridge converter on a 3 in $\times 2.74$ in circuit board.

\section{CONCLUSION}

A new integrated magnetic full wave topology is presented. The inductor winding is independent from the circuit voltage transfer function. The transformer can be designed to achieve a compromise between the number of inductor winding turns and the magnetic core size. The operation principle of the circuit is analyzed and the design relations are presented. Simulations and experiments verify the circuit analysis.

\section{APPENDIX}

\section{TRANSFORMER DESIGN FORMULA DERIVATION}

\section{A. Output Current Ripple}

The relation between the output current $I_{L}$ and the magnetomotive force on the gap $F_{g}$ is derived first. Mode I, as in Fig. 4, implies

$$
F_{p 1}=F_{s 1}-F_{I}-F_{I I I}-F_{g}+F_{L_{-} I} .
$$

Using the assumption that magnetomotive forces on the permeances of the core legs are neglected, i.e.

$$
F_{I} \approx 0, F_{I I I} \approx 0
$$

This leads to

$$
F_{p 1}=F_{s 1}-F_{g}+F_{L_{-} I} .
$$

For the same reason

$$
F_{p 2}=F_{g}-F_{L_{-} I} \text {. }
$$

Since $N_{p 1}=N_{p 2}$, and the currents in windings $N_{p 1}, N_{p 2}$ are the same, and therefore

$$
F_{p 1}=F_{p 2} \text {. }
$$

Also, from windings $N_{s 1}$ and $N_{L}$

$$
\begin{aligned}
F_{s 1} & =N_{s} I_{L} \\
F_{L_{-} I} & =N_{L} I_{L} .
\end{aligned}
$$

From (21)-(25)

$$
F_{g}=\left(N_{L}+\frac{N_{s}}{2}\right) I_{L} .
$$

By using the same method, (26) can also be derived in Mode II. So, (26) holds in the entire operating cycle.
Now integrating (9) in Mode II (or time duration $(0.5-D) T$ ), the peak-to-peak flux ripple in the gap is

$$
\Phi_{L_{-} d}=\frac{V_{o}(0.5-D)}{f_{s}\left(N_{L}+\frac{N_{s}}{2}\right)} .
$$

Since $\Phi_{L \_d}=F_{g_{-} d} P_{g}$, from (26) and (27), the peak-to-peak output current ripple is derived as

$$
I_{L_{-} d}=\frac{V_{o}(0.5-D)}{f_{s} P_{g}\left(N_{L}+\frac{N_{s}}{2}\right)^{2}} .
$$

This gives the criteria (12).

\section{B. Average Flux Density in The Center Leg}

Consider the average magnetomotive force on the gap

$$
F_{g_{-} a v}=\frac{B_{c_{-} a v} A_{c}}{P_{g}} .
$$

From (26) and (29)

$$
B_{c \_a v}=\frac{N_{L}+\frac{N_{s}}{2}}{A_{c}} I_{L_{-a v}} P_{g} .
$$

\section{Flux Swing}

In the center leg

$$
F_{g \_d}=\frac{B_{c_{-} d} A_{c}}{P_{g}} .
$$

From (26) and (30)

$$
B_{c_{-} d}=\frac{\left(N_{L}+\frac{N_{s}}{2}\right) I_{L_{-} d} P_{g}}{A_{c}} .
$$

In the outer leg, from (4-(6)

$$
\dot{\Phi}_{p 2}=\frac{2 V_{i}}{N_{p}}-\frac{\frac{V_{i} N_{L}}{N_{p}}+\frac{V_{o}}{2}}{N_{L}+\frac{N_{s}}{2}} .
$$

Integrating (31) in Mode I (or time duration DT) and considering (11) leads to

$$
B_{O_{-} d}=\frac{V_{o}\left(\frac{N_{L}}{N_{s}}+1-D\right)}{2 f_{s} A_{o}\left(N_{L}+\frac{N_{s}}{2}\right)} .
$$

This gives the criteria (16).

\section{ACKNOWLEDGMENT}

The authors wish to thank Dr. J. Sun, lead author of [2], for his professional ethics and courtesy in helping them avoid a potentially difficult situation, particularly for a graduate $\mathrm{Ph}$.D. student (together with his colleagues Dr. Sun simultaneously and independently developed the proposed circuit of this paper). After realizing that the circuits were the same, Dr. Sun immediately informed the authors and encouraged them to publish these results in both the IEEE TRANSACTIONS and in L. Yan's Ph.D. thesis. 


\section{REFERENCES}

[1] L. Yan, D. Qu, and B. Lehman, "Integrated magnetic full wave converter with flexible output inductor," Proc. IEEE APEC'02 Conf., pp. 824-830, 2002.

[2] J. Sun, K. F. Webb, and V. Mehrotra, "An improved current-doubler rectifier with integrated magnetics," Proc. IEEE APEC'02 Conf., pp. 831-837, 2002.

[3] E. Bloom, "New integrated-magnetic dc-dc power converter circuits \& systems," Proc. IEEE APEC'02 Conf., pp. 57-66, 1987.

[4] R. Severns and E. Bloom, Modern DC/DC Switchmode Power Converter Circuits. New York: Van Nostrand Reinhold Company, Dec. 1985

[5] E. Bloom, "Core selection for \& design aspects of an integrated-magnetic forward converter," Proc. IEEE APEC'86, pp. 141-150, 1986.

[6] D. K. Cheng, L. Wong, and Y. S. Lee, "Design, modeling, and analysis of integrated magnetics for power converters," Proc. IEEE PESC'00 Conf., pp. 320-325, 2000 .

[7] D. C. Hamill, "Lumped equivalent circuits of magnetic components: the Gyrator-capacitor approach," IEEE Trans. Power Electron., vol. 8, pp. 97-103, Apr. 1993

[8] _ - "Gyrator-capacitor modeling: a better way of understanding magnetic components," Proc. IEEE APEC'94 Conf., pp. 326-332, 1994

[9] M. E. Eaton, "Adding flux paths to SPICE's analytical capability improves the ease and accuracy of simulating power circuits," Proc. IEEE APEC'98 Conf., pp. 386-392, 1998.

[10] L. Yan and B. Lehman, "Better understanding and synthesis of integrated magnetics with simplified Gyrator model method," in Proc. IEEE PESC'01 Conf., 2001, pp. 433-438.

[11] G. Q. Morris, "Magnetically integrated full wave dc to dc converter," U.S. Patent 5555 494, Sept. 10, 1996.

[12] W. Chen, "Single magnetic low loss high frequency converter," U.S. Patent 5784 266, July 21, 1998.

[13] P. Xu, Q. Wu, P. Wong, and F. C. Lee, "A novel integrated current doubler rectifier," in Proc. IEEE APEC'00 Conf., 2000, pp. 735-740.

[14] F. D. Tan, J. L. Vollin, and S. M. Cuk, "A practical approach for magnetic core-loss characterization," IEEE Trans. Power Electron., vol. 10, pp. 124-130, Mar. 1995.

[15] W. G. Hurley, W. H. Wolfle, and J. G. Breslin, "Optimized transformer design: inclusive of high-frequency effects," IEEE Trans. Power Electron., vol. 13, pp. 651-659, July 1998.

[16] W. Song and B. Lehman, "Driving method for synchronous rectifier of dual-ended dc/dc converters," in Proc. HFPC'O0 Conf., 2000, pp. $446-451$

[17] N. Murakami, H. Namiki, K. Sakakibara, and T. Yachi, "A simple and efficient synchronous rectifier for forward dc-dc converters," in Proc. IEEE APEC'93 Conf., 1993, pp. 463-468.

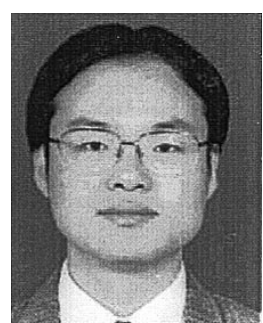

Liang Yan (S'01) was born in Jiangsu, China, in 1973. He received the B.E and M.E. degrees from Shanghai Jiaotong University, China, in 1995 and 1998, respectively, and is currently pursuing the Ph.D. degree in electrical engineering at Northeastern University, Boston.

From 1998 to 1999, he worked for Huawei Electrical, Inc., Shenzhen, China. His present research includes power electronics convert topologies, magnetic modeling, integrated magnetic transformer modeling, and topologies.

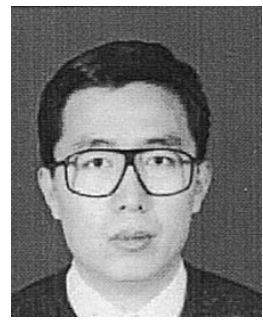

Dayu Qu (M'00) was born in Shandong, China, in 1970. He received the M.S. degree in electrical engineering in CPES from Virginia Polytechnic Institute and State University, Blacksburg, in 1999.

He then worked for Power-One, Inc., Boston, MA, as a Design Engineer. His research interests include power converter circuit topologies, power converter modeling and control, magnetic modeling, and EMI reduction in power converters.

Brad Lehman (M'92) received the B.E.E. degree from the Georgia Institute of Technology, Atlanta, in 1987, the M.S.E.E. degree from the University of Illinois at Champaign-Urbana, in 1988, and the Ph.D. degree in electrical engineering from the Georgia Institute of Technology, Atlanta, in 1992.

He is presently an Associate Professor and Associate Chair in the Department of Electrical and Computer Engineering, Northeastern University, Boston, MA, and previously was a Hearin Hess Distinguished Assistant Professor at Mississippi State University, Mississippi State, MS. He was a Visiting Scientist at the Massachusetts Institute of Technology, Cambridge. In 1999, he was a Science Advisor to the Commonwealth of Massachusetts, Science and Technology Committee (State Senate), for the Y2K issue in the Power Industry. He performs research in the areas of power electronics, electric motor drives, and control. A primary focus of his research is in the modeling, design and control of dc-dc converters.

Dr. Lehman received the Alcoa Science Foundation Fellowship. He was an NSF Presidential Faculty Fellow. He is an Associate Editor of the IEEE TRANSACTIONS ON POWER ElECTRONICS, and from 1993 to 1997, was an Associate Editor for the IEEE TRANSACTIONS ON AUTOMATIC CONTROL. 